\title{
Structure and Liquid Fragility in Sodium Carbonate
}

\author{
Mark Wilson* ${ }^{1}$ Mauro C.C. Ribeiro, ${ }^{2}$ Martin C. Wilding, ${ }^{3}$ Chris Benmore, ${ }^{4}$ \\ J.K.R. Weber, ${ }^{4,5}$ Oliver Alderman, ${ }^{4,5}$ Anthony Tamalonis, ${ }^{5}$ and J. B. Parise ${ }^{6}$ \\ ${ }^{1}$ Department of Chemistry, Physical and Theoretical Chemistry Laboratory, \\ University of Oxford, South Parks Road, Oxford OX1 3QZ, UK \\ ${ }^{2}$ Laboratório de Espectroscopia Molecular, Instituto de Química, \\ Universidade de Sāo Paulo, Av. Prof. Lineu Prestes 748, Sāo Paulo, 05508-000, Brazil. \\ ${ }^{3}$ Department of Chemistry, University College London, 20 Gordon Street, London, WC1H 0AJ, UK \\ ${ }^{4} X$-ray Science Division, Argonne National Laboratory, Argonne, IL60439, U.S.A. \\ ${ }^{5}$ Materials Development Inc, Arlington Heights, IL60004, U.S.A. \\ ${ }^{6}$ SUNY, Stony Brook, NY U.S.A.
}

The relationship between local structure and dynamics is explored for molten sodium carbonate. A flexible fluctuating-charge model, which allows for changes in the shape and charge distribution of the carbonate molecular anion, is developed. The system shows the evolution of highly temperaturedependent complex low-dimensional structures which control the dynamics (and hence the liquid fragility). By varying the molecular anion charge distribution the key interactions responsible for the formation of these structures can be identified and rationalised. An increase in the mean charge separation within the carbonate ions increases the connectivity of the emerging structures and leads to an increase in the system fragility. 


\section{INTRODUCTION.}

Potential relationships between system structure and dynamic behaviour are often difficult to characterise and untangle. Key to many theories is the concept of locally-favoured structures ${ }^{2-5}$ which may link with dynamic heterogeneities ${ }^{6-12}$. Systematic improvements in key experimental probes are allowing structure to be examined in more detail than ever before. However, key links between the time-averaged structure and the system dynamical behaviour often remain problematic ${ }^{2,13-15}$. Improvements in, for example, diffraction techniques (beam flux, sample levitation, pressure cells...) are generating information on systems over a wider range of conditions then previously possible. However, diffraction data still focusses on time- and spatially-averaged structure at a given state point. Additional information is required if important links are to be made between the underlying static structure and the system dynamics. Simulation models offer a clear pathway to linking structure and dynamics as the atom positions are known unequivocally and hence potentially complex inter-atomic spatial correlations may be untangled. Furthermore, the use of relatively simple descriptions of the inter-atomic interactions allows key parameters to be systematically varied and hence help isolate key controlling factors.

Molten carbonates have potentially significant roles in, for example, geochemistry ${ }^{16}, \mathrm{CO}_{2}$ sequestration $^{17}$ and fuel cells $^{18}$. As a result, understanding the structure and dynamics of these systems at an atomistic level is key. However, relatively few simulation models have been developed to date ${ }^{19-22}$.

Recent work has highlighted how the structure of molten sodium carbonate may be more complex than previously envisaged $^{23}$. Molecular ions are (historically) often considered as simple entities (effectively "large atoms"). Whilst this approximation is liable to be valid at high temperatures (for which the ions show significant rotational motion) there is a clear possibility that the geometry and charge distributions of these ions affects liquid structure (and hence the dynamics) as the system cools. Preliminary results, using models in which a fixed charge distribution is imposed on the molecular anions, indicate that the carbonate ions may closely associate, forming low-dimensional structures (see also simulation work on $\mathrm{CaCO}_{3}$ in an aqueous environment ${ }^{24}$ ). However, the factors which control the formation of these low-dimensional structures remains unclear. Furthermore, the effect of the formation of these structures on the system dynamics (in particular the ion diffusivities and related viscosities) is also unclear. Experimental viscosity data, obtained using oscillating crucible methods, are available ${ }^{25-27}$ but are inconsistent ${ }^{28}$. Models which impose a fixed charge distribution may be overly constrained as, in chemical terms, the distribution of charge over the atoms making up a molecular ion would be expected to be a function of the local environment.

\section{METHODS.}

In this Letter the evolution of the structure and dynamics in a key target system, molten sodium carbonate, is investigated both as a function of temperature and molecular charge distribution. To pursue this goal a fluctuating charge model (FCM) has been developed which employs both a flexible description of the $\mathrm{CO}_{3}^{2-}$ molecular anion and the internal charge distribution ${ }^{22,29-34}$. The use of a relatively simple description of the interatomic interactions (compared with, for example, employing large-scale electronic structure calculations) allows key model parameters to be systematically varied and, as a result, allows key interactions controlling the underlying structure and dynamics to be isolated. In a FCM the electronegativity, $\chi_{i \alpha}$, on an atomic site $\alpha$ within a given molecule $i$ is given by

$$
\chi_{i \alpha}=\chi_{i \alpha}^{0}+J_{\alpha \alpha}^{0} q_{i \alpha}+\sum_{\beta \neq \alpha} J_{\alpha \beta} q_{i \beta}+\sum_{j \neq i} \sum_{\beta} \frac{q_{j \beta}}{r},
$$

where $q_{i \alpha}$ is the charge on site $\alpha$ of ion $i, \chi_{i \alpha}^{0}$ is the electronegativity of atom $\alpha$ and $J_{\alpha \alpha}^{0}$ and $J_{\alpha \beta}$ control the intramolecular contributions to the charge distribution whilst the fourth term controls the inter-molecular atom-atom interactions. As a result, a single parameter, $\Delta \chi=\chi_{i C}^{0}-\chi_{i O}^{0}$, may be employed to effectively control the internal molecular charge distribution. The larger the value of $\Delta \chi$ the greater the charge separation on the oxygen and carbon atoms (termed $q_{O}$ and $q_{C}$ respectively). The charge separation can be quantified by defining $\Delta q=q_{C}-q_{O}$. Since the sum of the ion charges is constrained as $q_{C}+3 q_{O}=-2$ then $\Delta q=-2-4 q_{O} . \Delta q=0$ corresponds to an even spread of charge across the anion, $q_{O}=q_{C}=-1 / 2$. In the present work $\Delta q$ varies from $\Delta q \sim-0.7$ e to $\sim 2.5$ e (corresponding to $\left\{q_{O}, q_{C}\right\}=\{-0.33,-1\}$ e and $\{-1.12,1.36\}$ e respectively). Simulations are performed on systems containing 1296 atoms at fixed volume and temperature with the cell volumes obtained from the experimental densities ${ }^{35-37}$ and temperatures maintained using Nosé-Hoover thermostats ${ }^{38,39}$. 

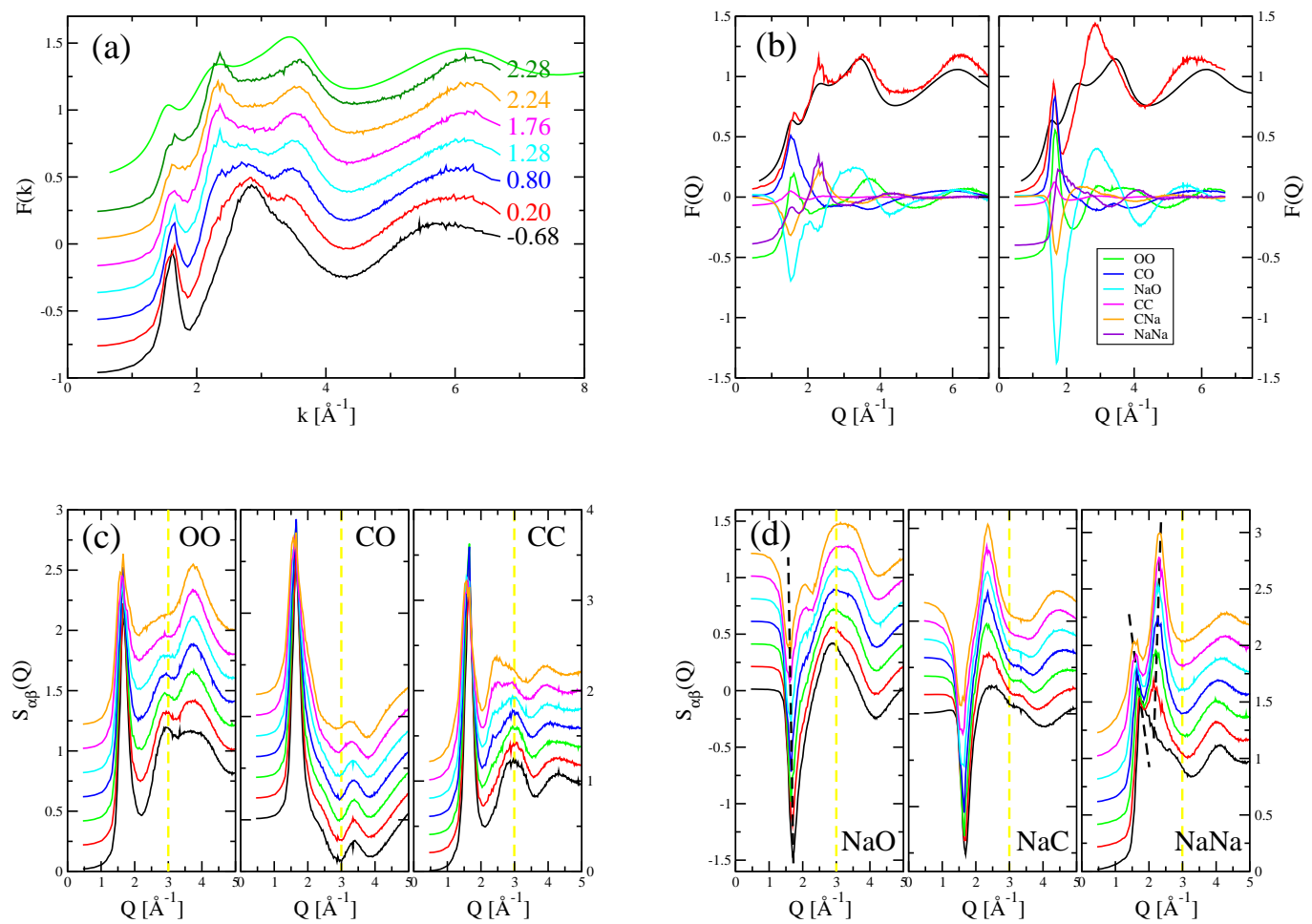

FIG. 1. (a) $F(Q)$ from HEXRD (light green topmost line) for $\mathrm{Na}_{2} \mathrm{CO}_{3}$ compared to that from the FCM at different values of $\Delta q$ as indicated. Successive curves are offset along the ordinate for clarity. (b) breakdowns of $F(Q)$ (black line - from HEXRD, red line - from simulation) into the weighted partial structure factors at the lowest and highest $\Delta q(\Delta q=-0.68 \mathrm{e}$ and $+2.48 \mathrm{e}$, left and right panels respectively) values respectively. (c) The evolution of $S_{O O}(Q), S_{C O}(Q)$ and $S_{C C}(Q)$ as a function of $\Delta q$. (d) The evolution of $S_{N a O}(Q), S_{N a C}(Q)$ and $S_{N a N a}(Q)$ as a function of $\Delta q$. In panels (a), (c) and (d) $\Delta q=-0.68,0.20,0.80,1.28,1.76,2.24$ and $2.28 \mathrm{e}$ (from bottom to top) and successive curves are offset along the ordinate for clarity.

\section{RESULTS AND DISCUSSION.}

Figure 1(a) shows the total structure factor, $F(Q)$, obtained by HEXRD experiment ${ }^{23}$ compared with the functions obtained from simulation using different values of $\Delta q . F(Q)$, is generated by combining the six Ashcroft-Langreth structure factors calculated directly from the Fourier components of the ion densities, $S_{\alpha \beta}(Q)=\left\langle A_{\alpha}^{*}(Q) A_{\beta}(Q)\right\rangle$, where $A_{\alpha}(Q)=\frac{1}{\sqrt{N_{\alpha}}} \sum_{j=1}^{N_{\alpha}} \exp \left(i \mathbf{Q} \cdot \mathbf{r}^{j}\right)$. As $\Delta q$ is increased (corresponding to greater charge separation in the carbonate anion) the agreement with experiment improves, in particular the intensity of the peak at low $-Q\left(Q \sim 1.6 \AA^{-1}\right)$ and the "filling in" between the peaks at $Q \sim 2.2 \AA^{-1}$ and $Q \sim 3.4 \AA^{-1}$. Figure 1 (b) shows the breakdown of $F(Q)$ into the six (X-ray weighted) partial structure factors for (b) low and (c) high $\Delta q$. The intensity of the peak at $Q \sim 1.6 \AA^{-1}$ is a superposition of all the partial structure factors. Figures $1(\mathrm{c})$ and (d) show the evolution of the six partial structure factors with changing $\Delta q$. There are a number of key changes in structure which contribute both to the intensity of the peak at $Q \sim 1.6 \AA^{-1}$ and the intensity in the range $\sim 2.2 \AA^{-1}<Q<\sim 3.4 \AA^{-1}$. $S_{N a N a}(Q)$ shows a "splitting" from a single peak at $Q \sim 1.8 \AA^{-1}$ (with a high- $Q$ shoulder) at low $\Delta q$, to a clear two-peak structure at high $\Delta q$ with peaks at $Q \sim 1.6 \AA^{-1}$ and $Q \sim 2.3 \AA^{-1}$. Analogous changes are observed in $S_{N a O}(Q)$ with the first peak shifting to lower $Q$ as $\Delta q$ increases coupled with the emergence of a feature at $Q \sim 2.3 \AA^{-1}$. In addition, the peak at $Q \sim 3 \AA^{-1}$ in $S_{N a O}(Q)$ shifts to higher $Q$ and broadens as $\Delta q$ increases, a change which contributes significantly to $F(Q)$ in the range $\sim 2.2 \AA^{-1}<Q<\sim 3.4 \AA^{-1} . S_{C C}(Q)$, although relatively low weighted in $F(Q)$, also shows significant changes as $\Delta q$ is increased.

Figure 2 shows the evolution in structure with changing $\Delta q$ in real space in the form of the six partial radial distribution functions at $T=1200 \mathrm{~K}$. As $\Delta q$ is increased a short length-scale emerges in $g_{C C}(r)$ at $r \sim 3.4 \AA$ with 

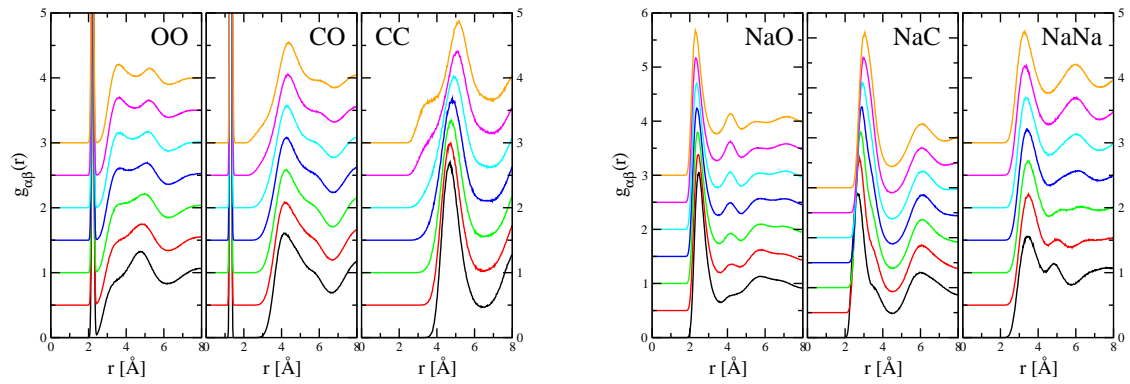

FIG. 2. Partial pair distribution functions, $g_{\alpha \beta}(r)$, as a function of $\Delta q$ as indicated with $\alpha \beta=\mathrm{OO}, \mathrm{CO}, \mathrm{NaO}, \mathrm{CC}, \mathrm{CNa}$ and NaNa. $\Delta q=-0.68,0.20,0.80,1.28,1.76,2.24$ and 2.28 e (from bottom to top) and successive curves are offset along the ordinate for clarity.
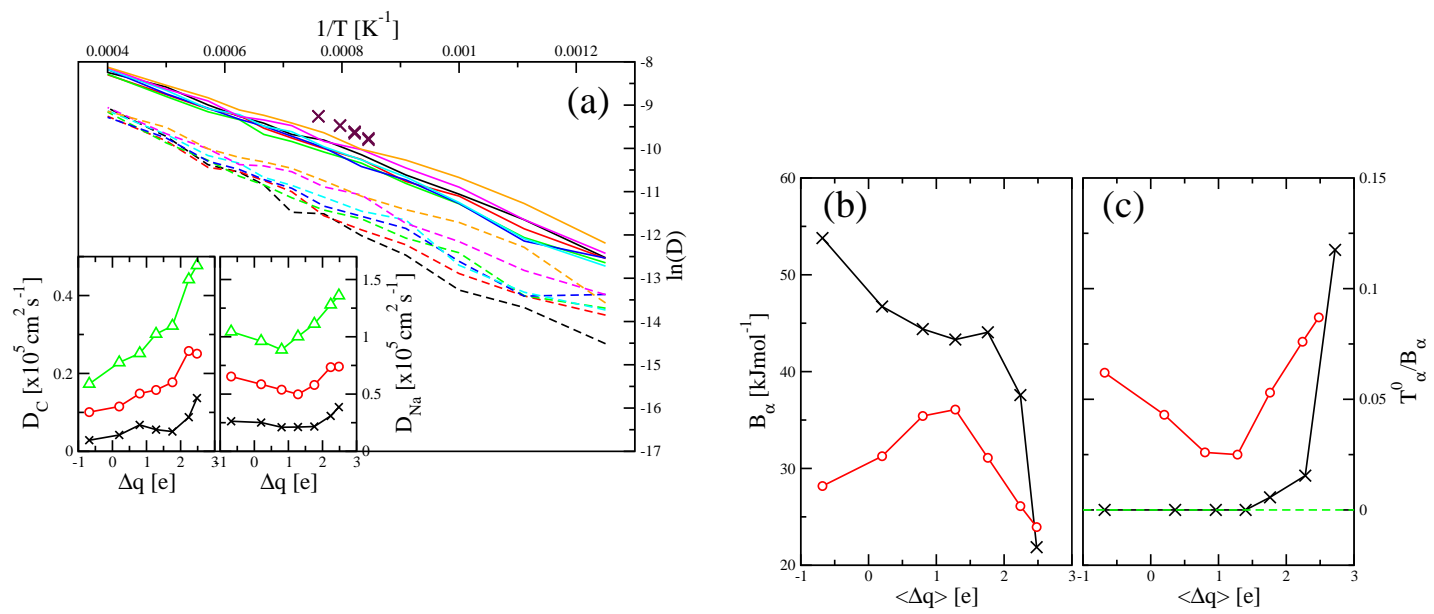

FIG. 3. (a) [Main panel]. Diffusion coefficients, $D_{\alpha}$, for $\alpha=\mathrm{C}$ (dashed lines) and $\alpha=\mathrm{Na}$ (solid lines) as a function of $T^{-1}$ for $\Delta q=-0.68,0.20,0.80,1.28,1.76,2.24$ and $2.28 \mathrm{e}$ (black, red, green, blue, cyan, magenta and orange lines respectively). The inset shows the respective diffusion coefficients as a function of $\Delta q$ at $T=1000 \mathrm{~K}, 1200 \mathrm{~K}$ and $1400 \mathrm{~K}$ (black, red and green lines respectively). (b) VTF coefficient $B_{\alpha}(\alpha=\mathrm{C}[\times], \mathrm{Na}[\mathrm{o}])$ as a function of $\Delta q$. (c) The ratio of the VTF coefficients $T_{\alpha}^{o} / B_{\alpha}$ $(\alpha=\mathrm{C}, \mathrm{Na})$ as a function of $\Delta q$.

the next-nearest length-scale moving to higher $r(r \sim 4.5 \longrightarrow 5.2 \AA)$. Over the same range the first peaks in $g_{N a O}(r)$ and $g_{N a C}(r)$ shift inward (from $r \sim 2.48 \longrightarrow 2.31 \AA$ ) and outward (from $r \sim 2.68 \longrightarrow 3.04 \AA$ ) respectively. Significant changes are also seen in $g_{N a N a}(r)$ with a small shift in the first peak (at $r \sim 3.6 \AA$ ) concomitant with a much larger shift in the position of the second peak (from $r \sim 5 \longrightarrow 6 \AA$ ).

Figure 3(a) shows the temperature-dependence of the diffusion coefficients (determined from the respective meansquared displacements) for the $\mathrm{C}$ and $\mathrm{Na}$ atoms $\left(D_{\alpha}\right.$ with $\alpha=\{\mathrm{C}, \mathrm{Na}\}$ respectively). At all temperatures the $\mathrm{Na}^{+}$ions show higher diffusivites than $\mathrm{CO}_{3}^{2-}$. The figure also shows the experimental sodium ion diffusivities ${ }^{40}$. The simulated values are in best agreement at high $\Delta q$ (for which the best agreement is shown with structural HEXRD data) although they are still smaller than those extracted experimentally. The inset shows the respective diffusion coefficients at three temperatures as a function of $\Delta q$. The carbon diffusion coefficients increase with charge separation whilst those for sodium show a more complex behaviour, falling for $\Delta q \lesssim 1$ but rising for $\Delta q \gtrsim 1$. To characterise any change in fragility the $\ln D_{\alpha} v .1 / T$ curves are fitted to a Vogel-Fulcher-Tammann (VFT) law of the form $D_{\alpha}=D_{\alpha}^{0} \exp \left[-B_{\alpha} /\left(T-T_{\alpha}^{0}\right)\right] . B_{\alpha}$ and $T_{\alpha}^{0}$ control the function curvature and hence characterize the fragility ${ }^{41,42}$. A value of $T_{\alpha}^{0}=0$ corresponds to a strong liquid (following an Arrhenius law) with $B_{\alpha}$ corresponding to the diffusive activation energy. Figure 3(b) shows the fitting parameter $B_{\alpha}$ as a function of $\Delta q$. For $\Delta q \lesssim 1 B_{C}$ and $B_{N a}$ show distinct behaviour (falling and rising with increasing $\Delta q$ respectively) whilst, for $\Delta q \gtrsim 1$, both functions fall as $\Delta q$ increases. Figure 3(c) shows the evolution of $T_{\alpha}^{0} / B_{\alpha}$ (a measure of the system fragility). Again, the $\mathrm{C}$ and Na sublattices show distinct behaviour. The carbonate diffusivities show a deviation from Arrhenius behaviour at $\Delta q \gtrsim 1.5$. The Na sublattice shows a deviation from Arrhenius behaviour both at low and high $\Delta q$ with a minimum at $\Delta q=1$. 
(a)
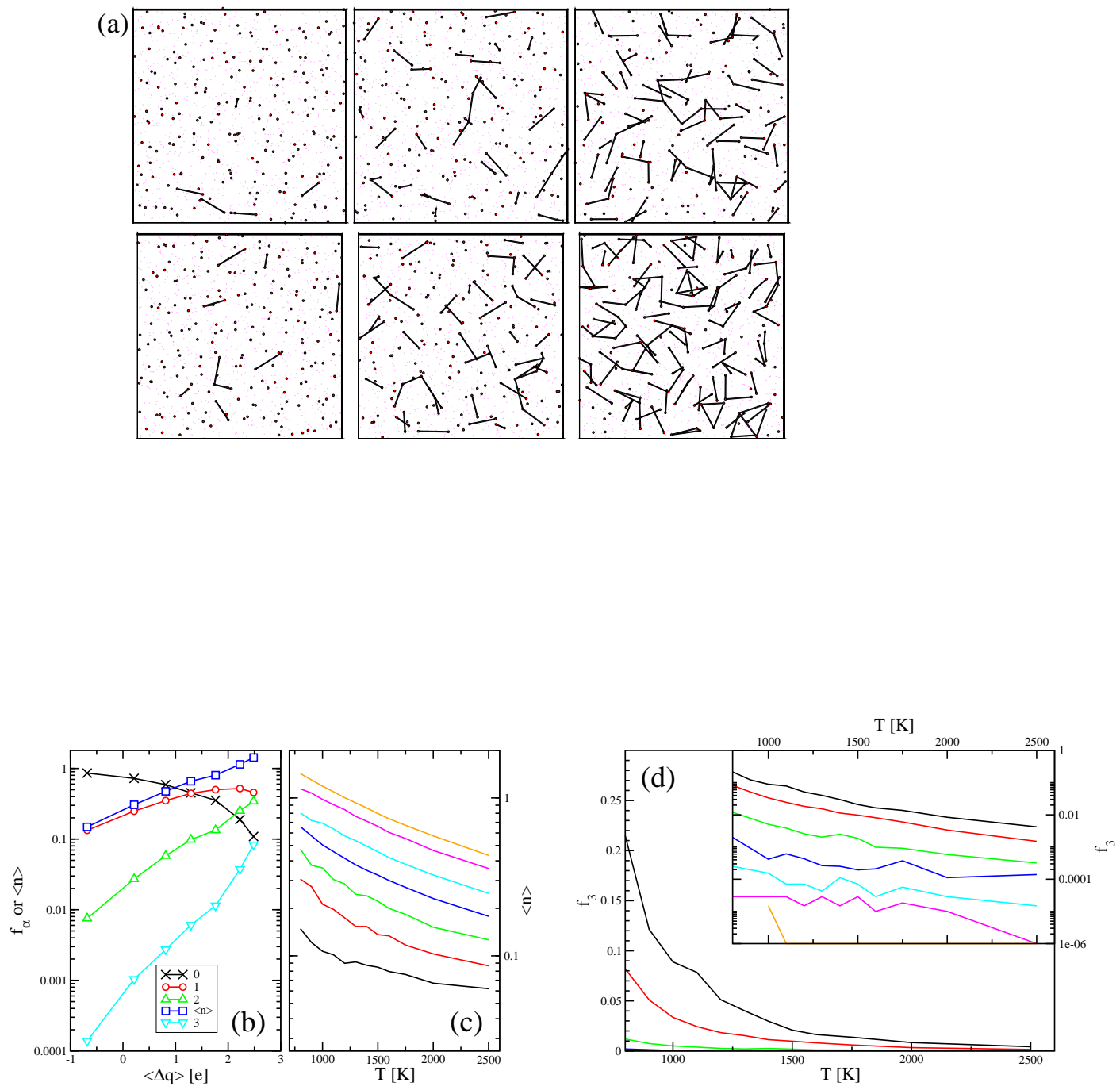

FIG. 4. (a) Molecular graphics "snapshots" of the $\mathrm{Na}_{2} \mathrm{CO}_{3}$ simulation cell at $T=1100 \mathrm{~K}$ (lower panels) and $T=1400 \mathrm{~K}$ (upper panels) showing the carbon atoms only for (from left to right) $\Delta q=-0.7,1.3$ and 2.5e. As $\Delta q$ increases (corresponding to a greater charge separation in the $\mathrm{CO}_{3}^{2-}$ anion) the number of complex low-dimensional structures increases. (b) Fraction of $n$-coordinate C-C "bonds" and the mean coordination number, $<n_{C C}>$, as a function of $\Delta q$ at $T=1200 \mathrm{~K}$. (c) The mean C-C coordination number, $\left\langle n_{C C}\right\rangle$, for different values of $\Delta q$ as a function of temperature. (d) Fraction of carbonate anions in $\left(\mathrm{CO}_{3}^{2-}\right)_{3}$ triangles as a function of both $\Delta q$ and $T$. The inset shows the same data on a logarithmic scale. In panels (c) and (d) $\Delta q=-0.68,0.20,0.80,1.28,1.76,2.24$ and $2.28 \mathrm{e}$ (black, red, green, blue, cyan, magenta and orange lines respectively).

The implication of the observations from figure 3 is that the carbonate and sodium ions diffusive behaviour becomes coupled at high $\Delta q$.

The pair distribution functions (figure 2) indicate that a short length-scale emerges in the carbon-carbon spatial correlations. Figure 4(a) shows a molecular graphics "snapshot" of this emerging structure as a function of $\Delta q$. At low charge separation these length-scales appear transient and are dominated by isolated C-C pairs. At intermediate $\Delta q$ these structures become longer-lived and significant numbers of (short) chains appear. At high $\Delta q$ longer chains form and more complex structural units, notably $\mathrm{C}_{3}$ rings and $\mathrm{C}_{4}$ tetrahedra. To properly quantify these structures 
figure 4(b) shows the distribution of C-C coordination numbers as a function of $\Delta q$ as well as the resulting mean coordination number, $\left\langle n_{C C}\right\rangle$. As $\Delta q$ increases the number of non-zero-coordinate sites increases concomitant with a fall in the number of isolated (0-coordinate) units. A similar change in structure is observed as the temperature is increased at fixed $\Delta q$. For example, figure 4 (c) shows how $\left\langle n_{C C}\right\rangle$ falls dramatically as the temperature is increased. Figure 4(d) shows the fraction of $\mathrm{C}_{3}$ rings formed as a function of $\Delta q$ and $T$. The number of these rings is a very strong function of both $\Delta q$ and $T$.

To further probe the emergent structures a number of additional spatial correlation functions are defined and shown in the supplementary information. Figure S1 shows a schematic to help explain the functions considered here. Figure S2 shows the three angular distribution functions $a(\theta), n(\alpha)$ and $r(\beta)$ as a function of $\Delta q$. The peaks at $\alpha \sim 0^{\circ}$ in $n(\alpha)$ indicate that pairs of carbonate ions showing a close C-C length-scale tend to "stack", the effect being stronger at high $\Delta q$ with the FWHM of the distributions reducing from $\Delta \alpha \sim 48^{\circ}$ to $\sim 24^{\circ}$ at low and high $\Delta q$ respectively. The evolution of $r(\beta)$ highlights that the relationship between $\mathrm{C}-\mathrm{O}$ bonds in these neighbouring carbonate ions changes from being staggered at low $\Delta q$ (showing a strong peak at $\beta \sim 180^{\circ}$ ) to eclipsed at high $\Delta q$ (showing strong peaks at $\beta \sim 0^{\circ}$ and $\sim 120^{\circ}$ ). $a(\theta)$ shows a change from favouring low angle peaks to favouring a peak at $\theta \sim 60^{\circ}$. These changes highlight a complex spatial relationship between the carbonate anions and the alkali cations. The most dramatic structure emerges in the carbonate anions themselves, with the formation of a range of low-dimensional structures including chains, triangles and tetrahedra. These structures are formed as a result of the strong electrostatic interactions between the negatively charged oxygen atoms and the positively charged alkali cations (as highlighted in the schematic figure S1. The moderation of the O-O interactions by the alkali cations results in the preferential formation of relatively close $\mathrm{CO}_{3}^{2-}-\mathrm{CO}_{3}^{2-}$ pairs in which the three $\mathrm{C}-\mathrm{O}$ bonds are eclipsed. These interactions also couple the $\mathrm{Na}^{+}$and $\mathrm{CO}_{3}^{2-}$ dynamics. As a result of the strong temperature-dependence of these structures the change in the underlying dynamics is reflected in the system fragility.

\section{SUMMARY AND CONCLUSIONS.}

In summary, liquid sodium carbonate shows the formation of highly temperature-dependent low-dimensional structures whose presence controls the system dynamics and hence controls the liquid fragility. The connectivities of these emerging structures are strongly correlated with the internal molecular anion charge distribution. As the charge separation increases the number of these low dimensional structures increases and results in an increase in the system fragility. Future work will consider how differing charge distributions is other important molecular ions (e.g. $\mathrm{NO}_{3}^{-}$, $\mathrm{NO}_{2}^{-}$etc) may correlate with system fragility. 


\section{SUPPORTING INFORMATION.}

The supporting information contains details on the angular correlation functions used to help interpret the formation of the emerging low-dimensional structures.

\section{ACKNOWLEDGEMENTS.}

Work by OA, AT, RW was supported under US Department of Energy grant number DE-SC0015241. This research used resources of the Advanced Photon Source, a U.S. Department of Energy (DOE) Office of Science User Facility operated for the DOE Office of Science by Argonne National Laboratory under Contract No. DE-AC02-06CH11357. This research used resources made available through a provostial support of the Joint Photon Sciences Institute at Stony Brook University. MW is grateful for support from the EPSRC Centre for Doctoral training, Theory and Modelling in Chemical Sciences, under grant EP/L015722/1. This paper conforms to the RCUK data management requirements. 
1 mark.wilson@chem.ox.ac.uk.

${ }^{2}$ Royall, C.; Kob, W. Locally favoured structures and dynamic length scales in a simple glass-former. J. Stat. Mech. 2017, 024001

3 Malins, A.; Eggers, J.; Tanaka, H.; Royall, C. Lifetimes and lengthscales of structural motifs in a model glassformer. Faraday Discuss. 2013, 167, 405.

4 Ronceray, P.; Harrowell, P. Favoured local structures in liquids and solids: a 3D lattice model. Soft Matter 2015, $11,3322$.

5 Russo, J.; Tanaka, H. Understanding water's anomalies with locally favoured structures. Nat. Comm. 2014, 5, 3556.

${ }^{6}$ Hurley, M.; Harrowell, P. Kinetic structure of a two-dimensional liquid. Phys. Rev. E 1995, 52, 1694.

7 Donati, C.; Douglas, J.; Kob, W.; Plimpton, S.; Poole, P.; Glotzer, S. Stringlike Cooperative Motion in a Supercooled Liquid. Phys. Rev. Lett. 1998, 80, 2338.

8 Ediger, M. Spatially Heterogeneous Dynamics in Supercooled Liquids. Annu. Rev. Phys. Chem. 2000, 51, 99.

9 Candelier, R.; Widmer-Cooper, A.; Kummerfeld, J.; Dauchot, O.; Biroi, G.; Harrowell, P.; Reichman, D. Spatiotemporal hierarchy of relaxation events, dynamical heterogeneities, and structural reorganization in a supercooled liquid. Phys. Rev. Lett. 2010, 105, 135702.

10 Pedersen, U.; Schroder, T.; Dyre, J.; Harrowell, P. Geometry of slow structural fluctuations in a supercooled binary alloy. Phys. Rev. Lett. 2010, 104, 105701.

11 de Souza, V.; Harrowell, P. Length scales of dynamic heterogeneities in a network of fluctuating mechanical constraints. Phys. Rev. E 2011, 83, 011501.

12 de Souza, V.; Harrowell, P. Structurally determined directionality identifies the boundary between mobile and immobile domains in a disordered material. J. Chem. Phys. 2012, 136, 054507.

13 Jack, R.; Dunleavy, A.; Royall, C. Information-Theoretic Measurements of Coupling between Structure and Dynamics in Glass Formers. Phys. Rev. Lett. 2014, 113, 095703.

${ }^{14}$ Ediger, M.; Harrowell, P. Perspective: Supercooled liquids and glasses. J. Chem. Phys. 2012, $137,080901$.

15 Wilson, M. Structure, dynamics and multiple length-scales in network-forming materials. J. Stat. Mech. 2016, 074010.

16 Jones, A.; Genge, M.; Carmody, L. Carbonate Melts and Carbonatites. Rev. Min. Geochem. 2013, $75,289$.

17 Corradini, D.; Coudert, F.-X.; Vuilleumier, R. Carbon dioxide transport in molten calcium carbonate occurs through an oxo-Grotthuss mechanism via a pyrocarbonate anion. Nat. Chem. 2016, 8, 454.

${ }^{18} \mathrm{Li}, \mathrm{X} . ; \mathrm{Xu}, \mathrm{N}$.; Zhang, L.; Huang, K. Low Temperature Densification and Electrical Property of a Carbonate-Added Proton Conducting Ceramic. ECS Trans. 2011, 35, 1267.

19 Tissen, J.; Janssen, G. Molecular-dynamics simulation of molten alkali carbonates. Mol. Phys. $1990,71,413$.

20 J.Habasaki, Molecular dynamics simulation of molten Li2CO3 and Na2CO3. Mol. Phys. 1990, 69, 115.

21 Corradini, D.; Coudert, F.-X.; Vuilleumier, R. Insight into the Li2CO3-K2CO3 eutectic mixture from classical molecular dynamics: Thermodynamics, structure, and dynamics. J. Chem. Phys 2016, 144, 104507.

22 Costa, M. Molecular dynamics of molten Li2CO3-K2CO3. J. Mol. Liq. 2008, $138,61$.

${ }^{23}$ Wilding, M. C.; Wilson, M.; Alderman, O. L. G.; Benmore, C.; Weber, J. K. R.; Parise, J. B.; Tamalonis, A.; Skinner, L. Low-Dimensional Network Formation in Molten Sodium Carbonate. Sci. Rep. 2016, 6, 24415.

24 Demichelis, R.; P.Raiteri,; J.D.Gale,; D.Quigley,; D.Gebauer, Stable prenucleation mineral clusters are liquid-like ionic polymers. Nat. Comm. 2011, 2, 590 .

25 Janz, G.; Saegusa, F. Molten Carbonates as Electrolytes: Viscosity and Transport Properties. J. Electro. Chem. Soc. 1963, $110,452$.

26 Sato, Y.; Yaegashi, S.; Kijima, T.; Takeuchi, E.; Tamai, K.; Hasebe, M.; Hoshi, M.; Yamamura, T. Viscosities of Molten Alkali Carbonates. Netsu Bussei 1999, 13, 156.

27 Sato, Y.; Yamazaki, T.; Kato, H.; Zhu, H.; Hoshi, M.; Yamamura, T. Viscosities of Li2CO3-Na2CO3 and Li2CO3-K2CO3 Binary Melts. Netsu Bussei 1999, 13, 162.

28 Nunes, V.; Lourenco, M.; Santos, F.; Nieto de Castro, C. Importance of Accurate Data on Viscosity and Thermal Conductivity in Molten Salts Applications. J. Chem. Eng. Data 2003, 48, 446.

29 Ribeiro, M. First sharp diffraction peak in the fragile liquid Ca0.4K0.6(NO3)1.4. Phys. Rev. B 2000, 61, 3297.

30 Ribeiro, M.; Almeida, L. Validating a polarizable model for the glass-forming liquid Ca0.4K0.6(NO3)1.4 by ab initio calculations. J. Chem. Phys. 2000, 113, 4722.

31 Ribeiro, M. Ionic dynamics in the glass-forming liquid Ca0.4K0.6(NO3)1.4: A molecular dynamics study with a polarizable model. Phys. Rev. B 2001, 63, 094205.

32 Rappe, A.; Goddard III, W. Charge equilibration for molecular dynamics simulations. J. Phys. Chem. 1991, 95, 3358.

${ }^{33}$ Liu, Y.-P.; Kim, K.; Berne, B.; Friesner, R.; Rick, S. Constructing ab initio force fields for molecular dynamics simulations. J. Chem. Phys. 1998, 108, 4739.

34 Rick, S.; Stuart, S.; Berne, B. Dynamical fluctuating charge force fields: Application to liquid water. J. Chem. Phys. 1994, $101,6141$.

35 Janz, G.; Dampier, F.; Lakshminarayanan, G.; Lorenz, P.; Tomkins, R. Molten Salts: Volume 1, Electrical Conductance, Density, and Viscosity Data. National Bureau of Standards Reference Data Series 1966, 15, 1.

36 Janz, G.; Krebs, U.; Siegenthaler, H.; Tomkins, R. Molten Salts: Volume 3 Nitrates, Nitrites, and Mixtures: Electrical Conductance, Density, Viscosity, and Surface Tension Data. J. Phys. Chem. Ref. Data 1972, 1, 581.

37 Janz, G. Thermodynamic and Transport Properties for Molten Salts. J. Phys. Chem. Ref. Data 1988, $17,1$. 
38 Nosé, S. A molecular dynamics method for simulations in the canonical ensemble. J. Chem. Phys. 1984, 81, 511.

39 Hoover, W. G. Canonical dynamics: Equilibrium phase-space distributions. Phys. Rev. A 1985, $31,1695$.

40 Spedding, P.; Mills, R. Trace-ion diffusion in molten alkali carbonates. J. Electro. Chem. Soc. $1965,112,594$.

41 Sastry, S. The relationship between fragility, configurational entropy and the potential energy landscape of glass-forming liquids. Nature 2001, 409, 164.

42 Binder, K.; Kob, W. Glassy Materials and Disordered Solids; World Scientific, Singapore, 2005. 


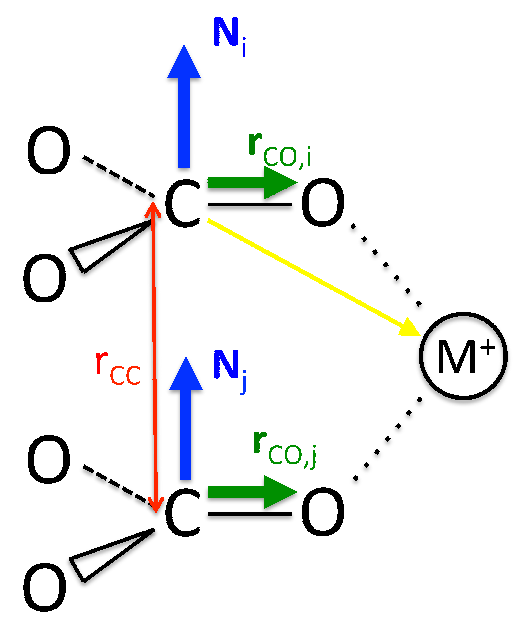

FIG. 5. TOC Graphic 